\title{
Strategy to avoid open surgical conversion after endovascular aortic aneurysm repair for patients with infrarenal abdominal aortic aneurysm
}

\author{
Byeoung-Hoon Chung', Seon-Hee Heo ${ }^{2,4}$, Yang-Jin Park ${ }^{2,4}$, Dong-lk Kim ${ }^{2,4}$, Duk-Kyoung Kim ${ }^{3,4}$, Young-Wook Kim ${ }^{5}$ \\ ${ }^{1}$ Department of Surgery, Jeonbuk National University Hospital, Jeonju, Korea \\ 2Division of Vascular Surgery, Department of Surgery, Samsung Medical Center, Sungkyunkwan University School of Medicine, \\ Seoul, Korea \\ ${ }^{3}$ Department of Internal Medicine, Samsung Medical Center, Sungkyunkwan University School of Medicine, Seoul, Korea \\ ${ }^{4}$ Cardiac and Vascular Center, Samsung Medical Center, Seoul, Korea \\ ${ }^{5}$ Division of Vascular Surgery, Department of Surgery, Kangbuk Samsung Hospital, Seoul, Korea
}

Purpose: Open surgical conversion (OSC) is the last treatment option for patients with endovascular aneurysm repair (EVAR) failure. We investigated the underlying causes of EVAR failure requiring OSC and attempted to determine strategies to avoid OSC after EVAR.

Methods: We retrospectively reviewed the database of patients who underwent OSC after EVAR from 2005 to 2018 in a single institution. Twenty-six OSCs were performed in 24 patients (median age, 74.5 years; $79.2 \%$ of males) who had undergone standard EVAR. We investigated pre-, intra-, and postoperative computed tomography or angiographic images and outcomes of the OSCs.

Results: Two main indications for OSC were persistent endoleak (50.0\%) and endograft infection (EI) (38.5\%). All 13 patients who underwent OSC due to endoleaks received EVAR outside of indications for use. Among 10 patients who underwent OSC due to El, we found overlooked infection sources in $7(70.0 \%)$ at the time of EVAR or during the surveillance period. OSC was performed at a median of 31.8 months (interquartile range, 9.4-69.8) after EVAR as an emergency (15.4\%) or elective (84.6\%) surgery. Aortic endograft was removed in $84.6 \%$ of cases (totally, 57.7\%; partially, $26.9 \%$ ), whereas it was preserved in 4 cases $(15.4 \%)$. After 26 OSCs, 2 early deaths $(7.7 \%)$ and 2 aortoenteric fistulae $(7.7 \%)$ developed as major complications.

Conclusion: OSC after EVAR was associated with relatively higher perioperative morbidity and mortality. To avoid OSC after EVAR, we recommend careful assessment of coexisting infection sources and avoidance of EVAR for patients with especially unfavorable anatomy for EVAR, particularly the in proximal neck.

[Ann Surg Treat Res 2020;99(6):344-351]

Key Words: Abdominal aortic aneurysm, Conversion to open surgery, Endovascular aneurysm repair

\section{INTRODUCTION}

According to the current practice guidelines [1], endovascular aneurysm repair (EVAR) has become the first treatment option for patients with infrarenal abdominal aortic aneurysm (AAA) when aortoiliac anatomy is suitable for EVAR. Based on previous
Received March 16, 2020, Revised August 11, 2020,

Accepted September 17, 2020

Corresponding Author: Young-Wook Kim

Division of Vascular Surgery, Department of Surgery, Kangbuk Samsung

Hospital, 29 Saemunan-ro, Jongno-gu, Seoul 03181, Korea

Tel: +82-2-2001-2569, Fax: +82-2-2001-2117

E-mail: young52.kim@samsung.com

ORCID: https://orcid.org/0000-0002-1106-3037
Copyright (c) 2020, the Korean Surgical Society

(c) Annals of Surgical Treatment and Research is an Open Access Journal. All articles are distributed under the terms of the Creative Commons Attribution NonCommercial License (http://creativecommons.org/licenses/by-nc/4.0/) which permits unrestricted non-commercial use, distribution, and reproduction in any medium, provided the original work is properly cited. 
large-scale, randomized, prospective studies, our current understanding regarding EVAR includes lower procedure-related mortality and higher reintervention rates compared with open surgical conversion (OSC), though early survival benefit was reported to diminish with time [2-4].

In current practice, the majority of post-EVAR complications can be managed with endovascular treatment. However, OSC can be the last treatment option for patients for whom endovascular treatment failed or was unavailable [5-7]. Though the number of OSCs following EVAR has decreased with the improvement of endovascular devices and techniques [7], a small number of patients still require OSC to treat complications after EVAR procedures. According to many previous reports $[6,8,9]$, OSC is technically demanding and carries significantly higher mortality and morbidity rates compared to those of elective standard OSC of the infrarenal AAA.

In the present study, we investigated the underlying causes of EVAR failures requiring OSC and attempted to determine a strategy to avoid OSC in EVAR patients.

\section{METHODS}

After approval from the Institutional Review Board (IRB) of Samsung Medical Center, we reviewed a database of patients who underwent OSC after standard EVAR at Samsung Medical Center between August 2005 and October 2018. The need for permission from the individual patients was waived for this study. Also, this retrospective study allowed the use of demographics and clinical characteristics of the patients by IRB (No. 2020-03-039).

OSC was defined as surgical opening of the aortic aneurysmal sac after EVAR regardless of removal of the aortic endograft. We excluded 3 primary OSCs during the EVAR procedure due to aortic rupture $(n=2)$ and access failure $(n=1)$ from this study.
For patients who underwent OSC, we investigated the indications and timing for OSC after EVAR. To determine the underlying causes of EVAR failure, we reviewed pre-, intra-, and postoperative CT or angiographic images, procedural details, and adjuvant procedures of EVAR. In addition to anatomical violation of device-specific indications for use (IFU) at the time of EVAR and late changes of aortoiliac anatomy and endograft device, we investigated periaortic infection sources by review of the pre- and post-EVAR CT images, history taking of febrile illness, trauma, or endovascular aortoiliac reintervention, or other invasive procedure around the time of detection of the endograft infection (EI). The type of endoleak was determined by reviewing the operative findings on OSC and imaging studies such as duplex ultrasonography, CT, and/or aortography.

For treatment of endoleak, we attempted endovascular treatment first when clinically available. In a patient presenting with symptoms or signs of AAA rupture, we performed emergency OSC without attempting endovascular treatment.

To establish the diagnosis of EI, we followed the diagnostic criteria of the Management of Aortic Graft Infection Collaboration (MAGIC) from the European Society of Vascular and Endovascular Surgery [10]. For patients suspected to have EI, we performed Gram stain, aerobic and anaerobic bacterial cultures, and cultures for fungus and Mycobacterium tuberculosis with blood and surgical specimens to identify infective organisms. For treatment of EI, we performed in situ aortoiliac reconstructions with cryopreserved arterial allograft (CAA) after removal of all infected stent grafts and aortic tissue. CAAs were sourced from our institutional tissue bank; the cryopreservation technique and surgical procedure of the aortic reconstruction have been previously described [11]. Fig. 1 shows in situ aortoiliac reconstruction with CAA after total explantation of the aortic endograft. Following aortic reconstruction with CAA, we implemented an empirical
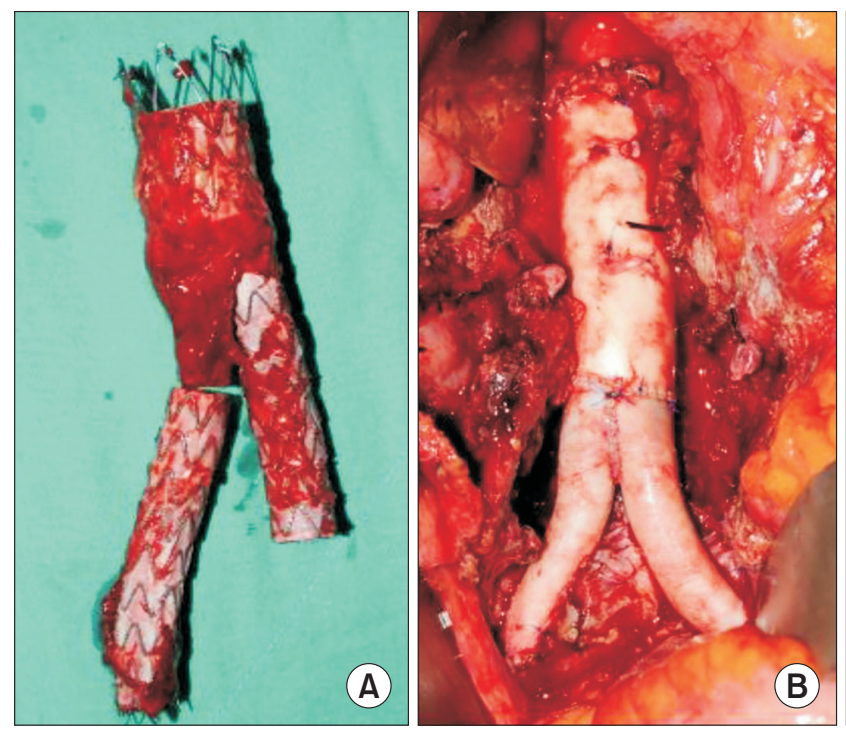

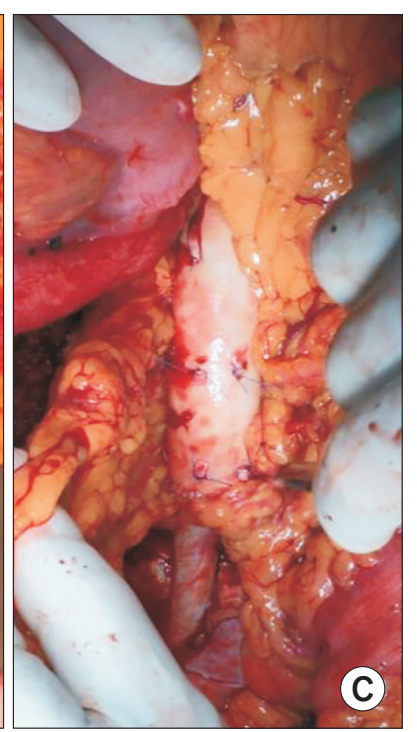

Fig. 1. Total explantation of aortic endograft and in situ aortoiliac reconstruction with a composite cryopreserved arterial allograft (CAA) for a patient with endograft infection at 71 months after endovascular aneurysm repair. (A) Explanted aortic endograft. (B) In situ aortoiliac reconstruction with a composite CAA. (C) Omental wrapping around the allograft. 
antibiotic therapy followed by selective therapy based on the bacterial culture and sensitivity test findings. For patients with EI, the duration of postoperative antibiotic use was at minimum 4 weeks of intravenous administration, followed by oral antibiotics until there was no clinical sign of infection and serum biologic markers (e.g., ESR, CRP, and WBC count) returned to normal levels. Infectious disease specialists were involved from the beginning of antibiotic therapy for all EI patients.

After hospital discharge, patients were periodically followed in the outpatient clinic every 3-6 months to check clinical symptoms/signs of infection, elevation of serum biologic markers (e.g., CRP, ESR, and WBC count), and contrast-enhanced CT images to detect periaortic infection and morphologic changes of the implanted CAA. To assess outcomes of OSCs, we reviewed early ( $\leq 30$ days) and late mortality and morbidity with suspected underlying causes.

Table 1. Characteristics of 24 patients who underwent open surgical conversion

\begin{tabular}{lc}
\hline \multicolumn{1}{c}{ Characteristic } & Data \\
Age $(y r)$ & $74.5(49-83)$ \\
Male sex & $19(79.2)$ \\
Coexisting disease & \\
$\quad$ Diabetes mellitus & $7(29.2)$ \\
COPD, moderate to severe & $5(20.8)$ \\
Coronary artery disease & $5(20.8)$ \\
Chronic renal failure on dialysis & $3(12.5)$ \\
Infection source at the time of EVAR & \\
Retroperitoneal abscess & $2(8.3)$ \\
Tuberculous osteomyelitis of lumbar spine & $1(4.2)$ \\
Infective AAA & $2(8.3)$ \\
$\quad$ Overlooked & $1(4.2)$ \\
After antibiotic therapy & $1(4.2)$ \\
\hline \hline
\end{tabular}

Values are presented as median (range) or number (\%). COPD, chronic obstructive pulmonary disease; EVAR, endovascular aneurysm repair; AAA, abdominal aortic aneurysm.

\section{RESULTS}

From August 2005 through October 2018, 26 OSCs were performed at Samsung Medical Center in 24 patients who had undergone standard EVAR to treat an infrarenal AAA. Nine patients (37.5\%) were institutional patients, and 15 patients $(62.5 \%)$ were transferred in from other institutions. In 2 patients, 2 OSCs were performed for each patient. Table 1 presents the characteristics of patients who underwent OSC after EVAR.

Five EVARs (20.8\%) were performed in patients with coexisting infection sources which included 2 infected AAAs (1 patient had an overlooked mycotic AAA at the time of EVAR and the other patient underwent an elective EVAR after 17 days of antibiotic therapy at the other institution) and 3 patients with periaortic infection source (psoas abscess $[n=2]$ and tuberculous osteomyelitis of lumbar vertebra $[\mathrm{n}=1]$ ) (Fig. 2). The patient with infected AAA who underwent EVAR following

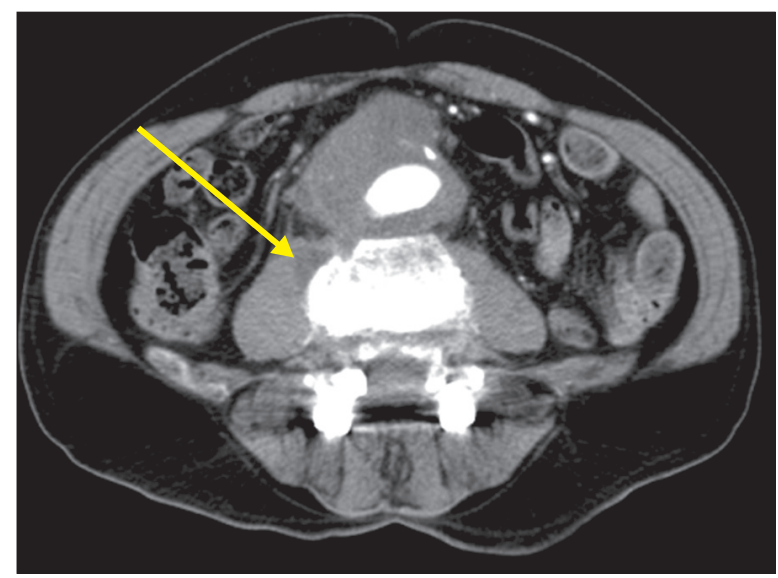

Fig. 2. A CT image before endovascular aneurysm repair (EVAR) shows abdominal aortic aneurysm and coexisting right psoas abscess (arrow) in a patient who underwent open surgical conversion at 28 months after EVAR.
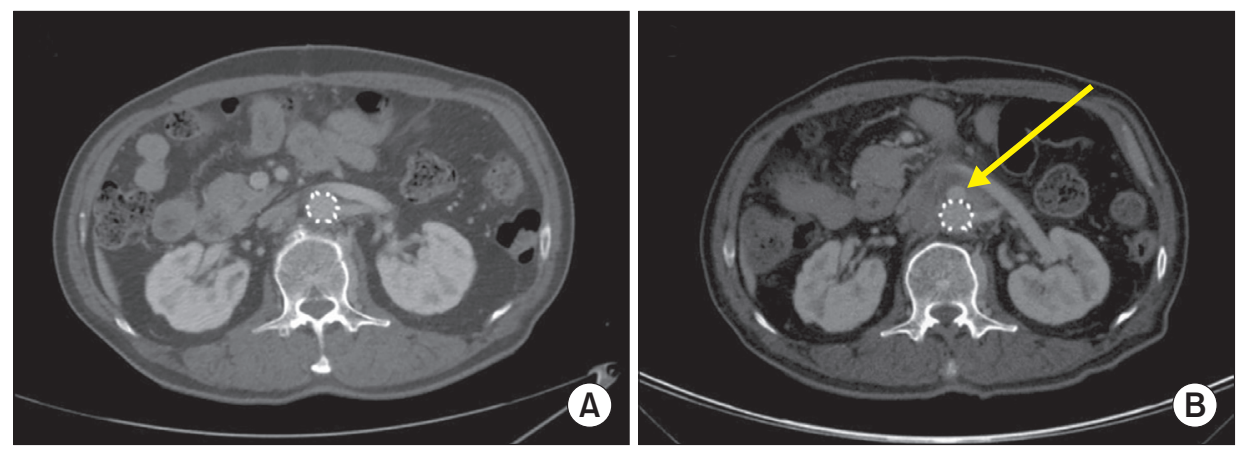

Fig. 3. The CT images in a patient with infected abdominal aortic aneurysm who underwent endovascular aneurysm repair (EVAR) after 17 days of antibiotic therapy at another hospital. (A) An axial CT image at the level of the left renal vein at 6 months after EVAR. (B) A new saccular aneurysm (arrow) at the anterior wall of the aorta at 7 months after EVAR, which displaced the left renal vein anteriorly. 
preoperative antibiotic therapy eventually developed a new small saccular aneurysm at the suprarenal aorta (Fig. 3) at 7 months after EVAR and presented to us with septic symptoms and signs.

In Table 2, we summarized preoperative aortoiliac anatomy and details of the primary EVAR and reintervention procedures. As shown in this table, $54.2 \%$ ( 13 of 24 patients) of EVARs were performed outside of device-specific IFU and all violated devicespecific IFU regarding the proximal neck.

The 2 most frequent indications for OSC were persistent endoleak ( 13 of $26,50.0 \%$ ) and EI (10 of $26,38.5 \%)$, followed by huge ( $>10 \mathrm{~cm}$ in diameter) progressive sac expansion of the AAA sac without endoleaks ( 3 of $26,11.5 \%$ ). When we researched the possible causes of EVAR failure requiring OSC, unfavorable aortoiliac anatomy and presence of an infection source either at the time of EVAR or after EVAR were the 2 most common causes (Table 3). Among patients with EI, there were 3 patients with history of calf cellulitis ( $\mathrm{n}=1$ ), bacterial pneumonia ( $\mathrm{n}$ $=1$ ), and dental procedure to treat gingival abscess $(n=1)$, in

Table 2. Details of aortoiliac anatomy and primary EVAR procedures in patients who underwent OSC

\begin{tabular}{lc}
\hline \multicolumn{1}{c}{ EVAR procedure } & Data $(\mathrm{n}=24)$ \\
\hline EVAR outside IFU (device-specific) & $13(54.2)$ \\
Proximal neck & $13(54.2)$ \\
$\quad$ Short neck $(<15 \mathrm{~mm})$ & 2 \\
Large neck $(>28 \mathrm{~mm})$ & 2 \\
$\quad$ Angled $>60^{\circ}, \leq 75^{\circ}$ & 6 \\
$\quad$ Reverse tapered $>20 \%$ & 7 \\
Iliac landing zone & $6(25.0)$ \\
$\quad$ Short CIA $(<15 \mathrm{~mm})$ & 1 \\
Large CIA (>25 mm) & 5 \\
Two or more anatomic risks & $8(33.3)$ \\
Endograft device & $10(41.7)$ \\
Endurant (Medtronic, Minneapolis, MN, USA) & $6(25.0)$ \\
Zenith (Cook Medical, Bloomington, IN, USA) & $4(16.7)$ \\
Excluder (Gore, Newark, DE, USA) & $3(12.5)$ \\
AneuRx (Medtronic, Minneapolis, MN, USA) & $1(4.2)$ \\
Domestic & $7(29.2)$ \\
Adjunctive procedure during EVAR & 4 \\
Hypogastric artery embolization and iliac limb & \\
$\quad$ extension & \\
Palmaz stent (Cordis Corp., Hialeah, FL, USA) at & 1 \\
the proximal neck & \\
Proximal extension cuff & 2 \\
Late endovascular reintervention to treat type II & $4(16.7)$ \\
endoleak & \\
Transfemoral arterial embolization & 2 \\
Translumbar AAA sac embolization & 2 \\
\hline \hline
\end{tabular}

Values are presented as number (\%) or number only. Numbers can be duplicated when they coexist.

EVAR, endovascular aneurysm repair; OSC, open surgical conversion; IFU, instruction for use; CIA, common iliac artery; AAA, abdominal aortic aneurysm. addition to 7 coexisting infections at the time of EVAR (Table 3).

Procedural details of OSCs are presented in Table 4. OSCs were performed at a median of 31.8 months (interquartile range [IQR], 9.4-69.8 months; range, 1-130 months) after EVAR urgent or emergency surgery in 4 cases (15.4\%). For OSC, suprarenal aortic cross clamping was required in 5 cases (19.2\%). Aortic endograft was removed totally ( 15 of $26,57.7 \%$ ) or partially $(7$ of 26, 26.9\%) while it was preserved in 4 patients (15.4\%). Partial explantation of endograft was usually performed in cases of endoleak, and total explantation $(n=10)$ of the endograft was performed for patients with EI. In patients with suprarenal fixing devices, a syringe technique was used to avoid aortic wall injury caused by the barbs of the fixing device.

We performed OSCs preserving aortic endograft for 4 cases with progressive sac enlargement without definite endoleak.

Table 3. Indications for 26 OSCs and suspected causes of EVAR failure

\begin{tabular}{|c|c|c|}
\hline Indication & Data & Suspected causes of EVAR failure \\
\hline Endoleak type & $13(50.0)$ & \\
\hline la & 5 & $\begin{array}{l}\text { Reverse tapered neck }(>20 \%)(\mathrm{n}=3) \\
\text { Severe }\left(>60^{\circ}\right) \text { angled proximal neck } \\
(\mathrm{n}=2) \\
\text { Short proximal neck }(<15 \mathrm{~mm})(\mathrm{n}=2) \\
\text { Large }(>28 \mathrm{~mm}) \text { proximal neck } \\
\quad \text { diameter }(\mathrm{n}=1)\end{array}$ \\
\hline $\mathrm{lb}$ & $2^{\text {a) }}$ & $\begin{array}{l}\text { Large }(>25 \mathrm{~mm}) \text { iliac diameter with } \\
\text { short }(<15 \mathrm{~mm}) \text { iliac landing zone }\end{array}$ \\
\hline IIla & 4 & $\begin{array}{l}\text { Late disconnection of iliac limb due to } \\
\text { progressive sac enlargement and } \\
\text { aortic remodeling }\end{array}$ \\
\hline IIIb & 2 & $\begin{array}{l}\text { Stitch hole bleeding from endograft } \\
(\mathrm{n}=1) \\
\text { Aortic rupture }(\mathrm{n}=1)\end{array}$ \\
\hline $\begin{array}{l}\text { Endograft } \\
\text { infection }\end{array}$ & $10(38.5)$ & \\
\hline $\begin{array}{l}\text { Coexisting } \\
\text { infection } \\
\text { source at } \\
\text { the time of } \\
\text { EVAR }\end{array}$ & 4 & $\begin{array}{l}\text { Infected AAA }(n=2) \\
\text { - Overlooked }(n=1) \\
\text { - After antibiotic therapy }(n=1) \\
\text { Psoas abscess }(n=1) \\
\text { Lumbar spine osteomyelitis }(n=1)\end{array}$ \\
\hline $\begin{array}{l}\text { Late, remote } \\
\text { infection } \\
\text { source }\end{array}$ & 3 & $\begin{array}{l}\text { Bacterial pneumonia }(n=1) \\
\text { Gingival abscess }(n=1) \\
\text { Calf cellulitis }(n=1)\end{array}$ \\
\hline AEF & $3^{\text {b) }}$ & Infection or bowel erosion $(\mathrm{n}=3)$ \\
\hline Others & $3(11.5)$ & $\begin{array}{l}\text { Progressive aortic sac enlargement } \\
\text { without endoleak or infection }\end{array}$ \\
\hline
\end{tabular}

Values are presented as number (\%) or number only.

OSC, open surgical conversion; EVAR, endovascular aneurysm repair; AAA, abdominal aortic aneurysm; AEF, aortoenteric fistula.

Sac enlargement denotes huge $(>10 \mathrm{~cm})$ and progressive aneurysm sac enlargement.

${ }^{a}$ Two patients with type Ib endoleak comprise 1 patient with combined type IIla endoleak and 1 patient with bilateral type Ib endoleak. ${ }^{\text {b) }}$ Three patients with AEF were double-counted for endograft infection and aortic rupture. 
All 4 patients showed progressive aneurysmal sac enlargement on follow-up CT images after sac obliteration surgery. One had aneurysmal sac size increased over $5.5 \mathrm{~cm}$, eventually requiring redo OSC.

Table 4. Procedural details of OSCs

\begin{tabular}{lc}
\hline \multicolumn{1}{c}{ Procedure } & Data $(\mathrm{n}=26)$ \\
\hline Timing after EVAR (mo) & $31.8(9.4-69.8)$ \\
& $43.7 \pm 37.4(1-130)$ \\
Clinical status & \\
Urgent or emergent & $4(15.4)$ \\
Elective & $22(84.6)$ \\
Aortic clamping & \\
Suprarenal & $5(19.2)$ \\
Infrarenal & $14(53.8)$ \\
Interrenal & $2(7.7)$ \\
None & $5(19.2)$ \\
Endograft removal & \\
Total & $15(57.7)$ \\
Partial & $7(26.9)$ \\
None & $4(15.4)$ \\
Aortoiliac reconstruction & \\
With prosthetic graft & $12(46.2)$ \\
With cryopreserved arterial allograft & $10(38.5)$ \\
Graft wrapping with omentum & $9(34.6)^{\mathrm{a})}$ \\
\hline
\end{tabular}

Values are presented as median (interquartile range), mean \pm standard deviation (range), or number (\%).

OSC, open surgical conversion; EVAR, endovascular aneurysm repair.

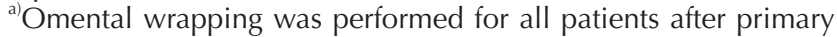
aortic reconstruction with cryopreserved allografts except a patient who had previously undergone radical total gastrectomy due to gastric cancer.
Table 5 demonstrates the early and late results of the 26 OSCs. There were 2 early postoperative deaths $(7.7 \%)$, which were due to sudden onset of hemoperitoneum on the postoperative day 12 and sudden onset hematemesis on the postoperative day 22 after OSCs. As an early surgical complication, rupture of the middle colic artery branch $(\mathrm{n}=1)$ and sigmoid colon ischemia combined with acute renal insufficiency $(n=1)$ developed.

Table 5. Results of 26 OSCs in 24 patients

\begin{tabular}{lc}
\hline \multicolumn{1}{c}{ Result } & Data \\
\hline Duration of follow-up (mo) & $9.4(1-111)$ \\
Loss to follow-up & $1(3.8)$ \\
Early ( $\leq 30$ days) outcome & \\
Death & $2(7.7)$ \\
Due to hematemesis & $1(3.8)^{\mathrm{a})}$ \\
Due to hemoperitoneum & $1(3.8)^{\mathrm{b})}$ \\
Complication & $3(11.5)$ \\
Rupture of middle colic artery branch & $1(3.8)^{\mathrm{c})}$ \\
Sigmoid colon ischemia & $1(3.8)$ \\
Acute renal insufficiency & $2(7.6)$ \\
Late (>30 days) outcome & $1(3.8)$ \\
Death & $2(7.7)$ \\
Aortoenteric fistula & \\
\hline \hline
\end{tabular}

Values are presented as median (range) or number (\%). Number can be overlapped due to double counting.

${ }^{a}$ One sudden death after hospital discharge occurred on the postoperative day 22 due to unidentified cause of hematemesis. ${ }^{b}$ Sudden hemoperitoneum developed on the postoperative day 12 after open surgical conversion (OSC) with aortoiliac reconstruction using cryopreserved allograft for a patient with endograft infection and aortoenteric fistula. ${ }^{\circ}$ Rupture of the midcolic arterial branch in a patient with type I neurofibromatosis occurred on the postoperative day 6 after OSC.

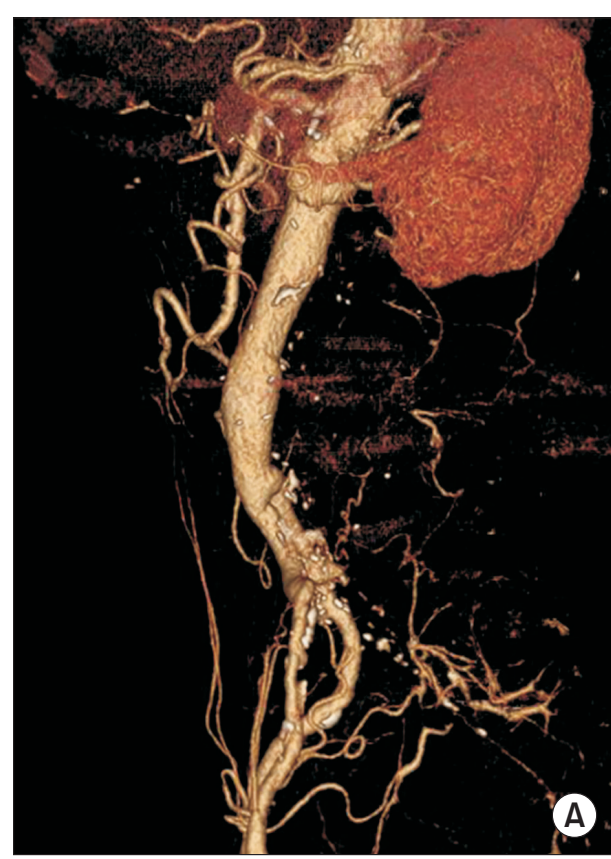

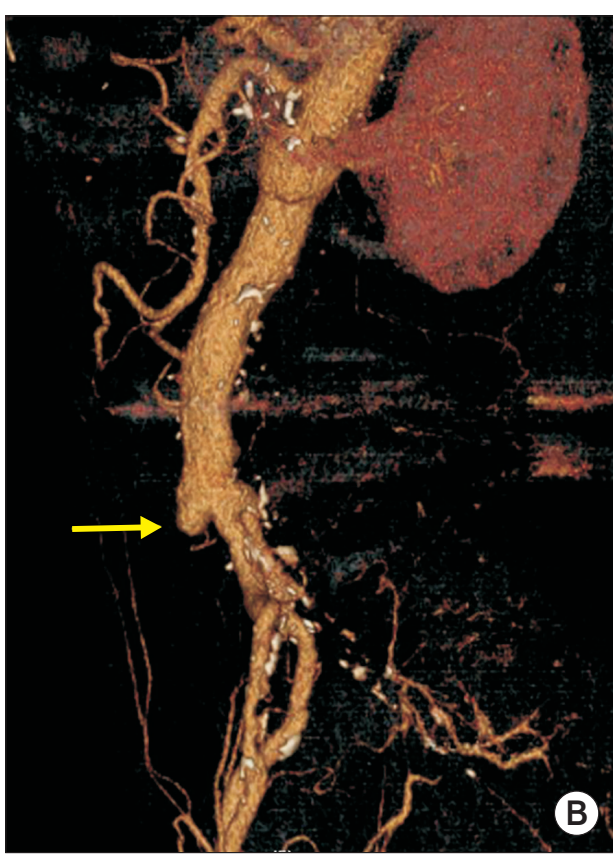

B
Fig. 4. Three-dimensional reformatted CT images in a patient who presented with aortoenteric fistula after open surgical conversion (OSC) due to endograft infection. (A) A CT image at 7 months after OSC (total explantation of the aortic endograft and in situ aortoiliac reconstruction with cryopreserved allograft. (B) A CT image at 27 months after OSC showing focal dilatation (arrow) of the cryopreserved arterial allograft. 
Rupture of the middle colic artery branch occurred in a patient with type I neurofibromatosis on the 6th day after OSC, which was successfully treated with endovascular coil embolization of the branch.

During the follow-up period (median, 9.4 months; IQR, 1.8-50.9 months; range, 1-130 months) after OSC, 2 aortoenteric fistulas (AEF) (7.7\%) developed as an aorta-related complication. One was detected at 27 months after an aortoiliac reconstruction with CAA for a patient with psoas abscess. There was sentinel gastrointestinal bleeding in the patient and focal dilatation of the CAA wall was detected on the follow-up CT image (Fig. 4). The patient was treated with redo OSC with CAA followed by antituberculous therapy. The other AEF developed in a patient who underwent OSC with preserving endograft and type IIIb endoleak. The patient developed AEF at 18 months after the first OSC. During the second OSC, we performed total explantation of the endograft and aortic reconstruction with CAA. This second OSC resulted in early postoperative death due to sudden development of hemoperitoneum on the postoperative day 12 .

There was 1 late death due to pneumonia at 5 months after OSC in a patient with hemodialysis-dependent chronic renal failure. The patient underwent OSC due to progressive, huge sac enlargement with persistent multiple type II endoleaks and consumption coagulopathy. For this patient, we controlled the type II endoleaks from inside of the sac and performed sac obliteration preserving endograft.

\section{DISCUSSION}

With the improvement of endovascular devices and endovascular technology, the frequency of EVAR failure was expected to decrease. However, the number of OSC has been increasingly reported in recent years $[7,12]$. This phenomenon can be explained by an increased cumulative frequency of EVAR-related complications with time and more aggressively performing EVAR for patients with especially unfavorable aortoiliac anatomy $[13,14]$. According to the National Surgical Quality Improvement Program (NSQIP) data of Ultee et al. [8], OSC showed significantly higher surgical mortality and morbidities than those of standard OSC of infrarenal AAA. Also, the variables of young age, female gender, non-white race, large aneurysmal diameter, and obesity were more frequently associated with OSC after EVAR.

The frequency of OSC has been reported around $2 \%$, ranging from 0.9 to $6 \%$ after standard EVAR $[5,6,8,9,15-20]$. The reported frequency of OSC may vary according to the duration of followup period and post-EVAR surveillance program as well as management strategy of the EVAR-related complications in each institution. Many recent review articles have suggested that OSC rates may be higher than reported when we take into account unreported cases of OSC [5,6,15-17].

In a meta-analysis of OSC after EVAR, Kouvelos et al. [12] reported that common indications for OSC were endoleak (62.4\%) and EI (9.5\%) [12]. Turney et al. [14] also reported that the main causes of OSC were type I or III endoleak, particularly in patients with hindering proximal neck anatomy. In our series, we have also experienced that endoleak and EI were the 2 main causes of OSC. As in previous reports [12,14,20], we found that unfavorable proximal neck anatomy was the main cause of endoleak requiring OSC. In current practice, specialized stent-graft devices and equipment are used to cope with unfavorable neck anatomy. However, there have been reported heterogeneous results regarding their effectiveness and longterm outcomes [21-23].

Among OSC patients, EVAR was performed outside the scope of anatomical IFU in $54.2 \%$ of our cases. When we consider the patients who underwent OSC due to endoleak, $90 \%$ of EVAR procedures were performed outside of IFU.

Among 10 patients who underwent OSC due to EI, we found coexisting infection sources in 7 patients $(70.0 \%$ ) which included 4 at the time of EVAR and 3 during the followup period on our retrospective investigation. Sufficient preoperative evaluation for infection sources could reduce the possibility of postprocedural EI. Furthermore, late and remote sources of infection can also be prevented or better managed through the proper use of prophylactic antibiotics.

Aortic rupture including AEF were also indications for OSC. OSCs were performed with $(n=22)$ or without $(n=4)$ endograft removal. We removed total endografts for all patients with EI and partially removed in patients with endoleak patients. AAA sac opening without removal of endograft was possible by performing control of endoleaks and obliteration of aneurysm sac for 4 patients with progressive sac enlargement without definite endoleak $(n=3)$ and type IIIb endoleak $(n=1)$. Those patients showed progressive aneurysmal sac enlargement and redo OSC was eventually required in 1 patient. We thought that AAA sac obliteration only cannot prevent the progression of aneurysm sac enlargement.

An OSC procedure requiring endograft removal can be divided into 3 parts; aortic clamping and sac opening, endograft removal, and aortoiliac reconstitution. Among these procedures, endograft removal was the most challenging for us, especially in patients with suprarenal fixing endograft. During total explantation of suprarenal fixing stent graft, we used a syringe technique to reduce suprarenal aortic wall injury by the barbs on the fixing struts [24]. To avoid aortic wall injury by the sharp cut edge of the syringe, we used an electric bone saw to cut the syringe and bone file to smoothen the cut edge of the syringe.

The aortic wall in contact with aortic endograft for long durations is characterized by thinning and inflammation of the aortic wall with periaortic adhesion. An overzealous attempt 
of total explantation may cause serious pararenal aortic wall injury, which may result in an intra- or postoperative disaster $[18,24,25]$. Therefore, part of the endograft can be preserved if it is not infected. In a patient who developed hemoperitoneum in the early postoperative period after OSC, we had difficulties in removing the endograft with the syringe technique. Though we were unable to confirm the exact source of postoperative bleeding, intraoperative aortic injury is suspected as a cause of intraperitoneal bleeding with CAA rupture.

All implanted aortic prostheses are at risk for infection either at the time of implantation or later by way of hematogenous seeding. According to a report by Schermerhorn et al. [26], based on analysis of more than 45,000 Medicare beneficiaries, frequency of EI or AEFs at 4 years after EVAR was similar to those after OSC of AAA. While aortic graft infection is usually presented at an average of 3 years or later postoperatively, EI after EVAR often manifests earlier in graft infection after open surgery for reasons that remain unclear [27,28]. In our series, EI was detected at a median of 29.5 months (IQR, 7.1-74 months) after EVAR.

The American Heart Association has recommended the use of prophylactic antibiotics before dental procedures in patients with a prosthetic cardiac valve, previous bacterial endocarditis, or cardiac transplant with valve regurgitation [29]. However, there had been no guidelines or recommendations for prophylactic use of antibiotics in patients with aortic endografts until the new practice guidelines of the Society for Vascular Surgery were released in 2018 [1]. According to these guidelines [1], appropriate antibiotic prophylaxis is recommended in patients with an aortic prosthesis who are undergoing any dental procedure or other invasive procedure involving the respiratory, gastrointestinal, or genitourinary tracts, particularly in immunocompromised patients.

As for the limitations of our retrospective study, we have to accept the possibility of selection bias due to the referral pattern of patients and local unavailability of the endovascular device in Korea to treat persistent endoleak.

In conclusion, the 2 most common indications for OSC after EVAR were endoleak and EI. After OSC, we have experienced relatively higher rates of surgical mortality and morbidities. By this retrospective review of underlying causes of EVAR failure for patients requiring OSC, we confirmed that some of them were preventable by proper selection of EVAR candidates. To avoid OSC after EVAR, we recommend not performing EVAR for patients with severe unfavorable neck anatomy or coexisting infection sources.

\section{ACKNOWLEDGEMENTS}

\section{Conflict of Interest}

No potential conflict of interest relevant to this article was reported.

\section{ORCID iD}

Byeoung-Hoon Chung: https://orcid.org/0000-0002-2737-1261

Seon-Hee Heo: https://orcid.org/0000-0002-0250-2229

Yang-Jin Park: https://orcid.org/0000-0001-8433-2202

Dong-Ik Kim: https://orcid.org/0000-0003-0674-8725

Duk-Kyoung Kim: https://orcid.org/0000-0002-2348-8948

Young-Wook Kim: https://orcid.org/0000-0002-1106-3037

\section{Author Contribution}

Conceptualization: BHC, YWK

Formal Analysis: BHC, DIK

Investigation: BHC, SHH, YJP

Methodology: DKK

Project Administration: BHC, YWK

Writing - Original Draft: BHC, SHH, YJP

Writing - Review \& Editing: DIK, DKK, YWK

\section{REFERENCES}

1. Chaikof EL, Dalman RL, Eskandari MK, Jackson BM, Lee WA, Mansour MA, et al. The Society for Vascular Surgery practice guidelines on the care of patients with an abdominal aortic aneurysm. J Vasc Surg 2018;67:2-77.

2. EVAR Trial Participants. Endovascular aneurysm repair versus open repair in patients with abdominal aortic aneurysm (EVAR trial 1): randomised controlled trial. J Vasc Surg 2005:42:592.

3. Patel R, Sweeting MJ, Powell JT, Greenhalgh RM; EVAR trial investigators. Endovascular versus open repair of abdominal aortic aneurysm in 15-years' follow-up of the UK endovascular aneurysm repair trial 1 (EVAR trial 1): a randomised controlled trial. Lancet 2016;388:2366-74.

4. Greenhalgh RM, Brown LC, Kwong GP,
Powell JT, Thompson SG; EVAR trial participants. Comparison of endovascular aneurysm repair with open repair in patients with abdominal aortic aneurysm (EVAR trial 1), 30-day operative mortality results: randomised controlled trial. Lancet 2004:364:843-8.

5. Millon A, Deelchand A, Feugier P, Chevalier JM, Favre JP; University Association for Research in Vascular 
Surgery (AURC). Conversion to open repair after endovascular aneurysm repair: causes and results. A French multicentric study. Eur J Vasc Endovasc Surg 2009:38:429-34.

6. Moulakakis KG, Dalainas I, Mylonas S, Giannakopoulos TG, Avgerinos ED, Liapis $\mathrm{CD}$. Conversion to open repair after endografting for abdominal aortic aneurysm: a review of causes, incidence, results, and surgical techniques of reconstruction. J Endovasc Ther 2010;17:694-702.

7. Harris PL, Vallabhaneni SR, Desgranges P, Becquemin JP, van Marrewijk C, Laheij RJ. Incidence and risk factors of late rupture, conversion, and death after endovascular repair of infrarenal aortic aneurysms: the EUROSTAR experience. European Collaborators on Stent/graft techniques for aortic aneurysm repair. J Vasc Surg 2000;32:739-49.

8. Ultee KH, Soden PA, Zettervall SL, Darling J, Verhagen HJ, Schermerhorn ML. Conversion from endovascular to open abdominal aortic aneurysm repair. J Vasc Surg 2016;64:76-82.

9. Terramani TT, Chaikof EL, Rayan SS, Lin PH, Najibi S, Bush RL, et al. Secondary conversion due to failed endovascular abdominal aortic aneurysm repair. J Vasc Surg 2003:38:473-7.

10. Lyons OT, Baguneid M, Barwick TD, Bell RE, Foster N, Homer-Vanniasinkam S, et al. Diagnosis of aortic graft infection: a case definition by the management of aortic graft infection collaboration (MAGIC). Eur J Vasc Endovasc Surg 2016;52:758-63.

11. Heo SH, Kim YW, Woo SY, Park YJ, Kim DK, Chung DR. Recent results of in situ abdominal aortic reconstruction with cryopreserved arterial allograft. Eur J Vasc Endovasc Surg 2017;53:158-67.

12. Kouvelos G, Koutsoumpelis A, Lazaris A, Matsagkas M. Late open conversion after endovascular abdominal aortic aneurysm repair. J Vasc Surg 2015;61:1350-6.

13. Georgiadis GS, Trellopoulos G, Antoniou GA, Gallis K, Nikolopoulos ES, Kapoulas $\mathrm{KC}$, et al. Early results of the Endurant endograft system in patients with friendly and hostile infrarenal abdominal aortic aneurysm anatomy. J Vasc Surg 2011:54:616-27.

14. Turney EJ, Steenberge SP, Lyden SP, Eagleton MJ, Srivastava SD, Sarac TP, et al. Late graft explants in endovascular aneurysm repair. J Vasc Surg 2014:59:88693.

15. Jimenez JC, Moore WS, Quinones-Baldrich WJ. Acute and chronic open conversion after endovascular aortic aneurysm repair: a 14-year review. J Vasc Surg 2007;46:6427.

16. van Marrewijk CJ, Fransen G, Laheij RJ, Harris PL, Buth J; EUROSTAR Collaborators. Is a type II endoleak after EVAR a harbinger of risk? Causes and outcome of open conversion and aneurysm rupture during follow-up. Eur J Vasc Endovasc Surg 2004:27:128-37.

17. Lifeline Registry of EVAR Publications Committee. Lifeline registry of endovascular aneurysm repair: long-term primary outcome measures. J Vasc Surg 2005:42:1-10.

18. Klonaris C, Lioudaki S, Katsargyris A, Psathas E, Kouvelos G, Doulaptsis M, et al. Late open conversion after failed endovascular aortic aneurysm repair. J Vasc Surg 2014:59:291-7.

19. Kelso RL, Lyden SP, Butler B, Greenberg RK, Eagleton MJ, Clair DG. Late conversion of aortic stent grafts. J Vasc Surg 2009;49:589-95.

20. Wu Z, Xu L, Qu L, Raithel D. Seventeen years' experience of late open surgical conversion after failed endovascular abdominal aortic aneurysm repair with 13 variant devices. Cardiovasc Intervent Radiol 2015:38:53-9.

21. Gallitto E, Gargiulo M, Freyrie A, Bianchini Massoni C, Pini R, Mascoli C, et al. Results of standard suprarenal fixation endografts for abdominal aortic aneurysms with neck length $\leq 10 \mathrm{~mm}$ in high-risk patients unfit for open repair and fenestrated endograft. J Vasc Surg 2016;64:563-70.

22. Malas MB, Hicks CW, Jordan WD Jr, Hodgson KJ, Mills JL Sr, Makaroun
MS, et al. Five-year outcomes of the PYTHAGORAS U.S. clinical trial of the Aorfix endograft for endovascular aneurysm repair in patients with highly angulated aortic necks. J Vasc Surg 2017;65:1598-607.

23. Jordan WD Jr, Mehta M, Varnagy D, Moore WM Jr, Arko FR, Joye J, et al. Results of the ANCHOR prospective, multicenter registry of EndoAnchors for type Ia endoleaks and endograft migration in patients with challenging anatomy. J Vasc Surg 2014;60:885-92.

24. Usatii A, Payne W, Santilli S. Removal of an infected aortic endograft and open aortic reconstruction: technical remarks. Ann Vasc Surg 2013;27:679-83.

25. Matsagkas M, Kouvelos GN, Peroulis M. Safe and fast proximal aortic control using an aortic balloon through direct graft puncture for the explantation of an abdominal endograft with suprarenal fixation. Interact Cardiovasc Thorac Surg 2014:18:519-21.

26. Schermerhorn ML, O'Malley AJ, Jhaveri A, Cotterill P, Pomposelli F, Landon BE. Endovascular vs, open repair of abdominal aortic aneurysms in the Medicare population. N Engl J Med 2008;358:464-74.

27. Murphy EH, Szeto WY, Herdrich BJ, Jackson BM, Wang GJ, Bavaria JE, et al. The management of endograft infections following endovascular thoracic and abdominal aneurysm repair. J Vasc Surg 2013:58:1179-85.

28. Fatima J, Duncan AA, de Grandis E, Oderich GS, Kalra M, Gloviczki P, et al. Treatment strategies and outcomes in patients with infected aortic endografts. J Vasc Surg 2013;58:371-9.

29. Nishimura RA, Otto CM, Bonow RO, Carabello BA, Erwin JP 3rd, Fleisher LA, et al. 2017 AHA/ACC focused update of the 2014 AHA/ACC Guideline for the Management of Patients With Valvular Heart Disease: a report of the American College of Cardiology/American Heart Association Task Force on Clinical Practice Guidelines. Circulation 2017;135:e1159-95. 